Umstellen auf generisches Statin

\title{
Häufig steigt dann das Infarktrisiko
}

\section{Beim Umstellung vom Original auf ein generisches Statin werden häufig nicht äquipotente, sondern zu nied- rige Statindosen verordnet. Die Folge ist ein Anstieg des Infarktrisikos.}

- Statin sei gleich Statin, aus Kostengründen sind möglichst generische Cholesterinsenker zu verordnen, so lautet die Vorgabe der Gesundheitspolitik in immer mehr Ländern. In den Niederlanden müssen Ärzte seit 2009 begründen, wenn sie ein Originalstatin verordnen wollen. Daraufhin wurden in den ersten drei Monaten des Jahres fast 40000 Herzpatienten von Atorvastatin auf generisches Simvastatin umgestellt.

Mithilfe einer zentralen Pharma-Datenbank, die nahezu alle Verordnungen des Landes erfasst, konnte untersucht werden, wie genau umgestellt wurde.
Dabei zeigte sich: Bei einem Drittel der Patienten wurde nicht berücksichtigt, dass Atorvastatin stärker wirksam ist als Simvastatin, die Patienten erhielten eine geringere Statindosis als zuvor. Über die Gesamtgruppe der knapp 40000 Patienten errechnete das Team einen durchschnittlichen LDL-Anstieg von 5-6\%. Die müsste nach den Ergebnissen einschlägiger Metaanalysen der großen Statinstudien einen etwa 3\%igen Anstieg von Herzinfarkten und Schlaganfällen zur Folge haben. Dem kurzfristigen Spareffekt bei den Statinkosten folgen demnach schon bald Mehrausgaben für Akutbehandlung und Rehabilitation von Infarkten und Insulten.

$\mathrm{DE}$.

- Quelle: D. Liew et al., ESC-Jahreskongress 2010; Eur Heart J Vol. 31, Abstract Supplement, \#3562

\section{Kurz notiert}

Vorhofflimmern Sartane wirkungslos Prospektive Studien halten nicht immer das, was Metaanalysen versprechen. In mehreren Studien mit $\mathrm{AT}_{1}$-Blockern zeigten sich in den Verumgruppen signifikante Reduktionen der Inzidenz des Vorhofflimmerns, Metaanalysen legten ein therapeutisches Potenzial nahe. Nun zeigte eine Prospektivstudie des deutschen Kompetenznetzes Vorhofflimmern, dass Sartane nicht antiarrhythmisch wirksam sind. Dies berichtete Studienleiter Prof. A. Götte, Paderborn. Die ANTIPAF-Studie untersuchte 422 Patienten mit paroxysmalem Vorhofflimmern ohne strukturelle Herzkrankheit. Studienmedikation war Olmesartan 40 $\mathrm{mg} / \mathrm{d}$. Ein Jahr lang übermittelten die Studienteilnehmer mithilfe eines Chipkarten-EKGs täglich mindestens ein EKG. Nach Analyse von 87818 Tele-EKGs zeigte sich: kein Unterschied zwischen Sartan und Placebo.

- A. Götte et al., ESC-Jahreskongress 2010; Eur Heart J, Vol. 31, Abstr Suppl, \#3772

\section{Patient mit Schuppenflechte}

\section{Achten Sie auf sein Herz!}

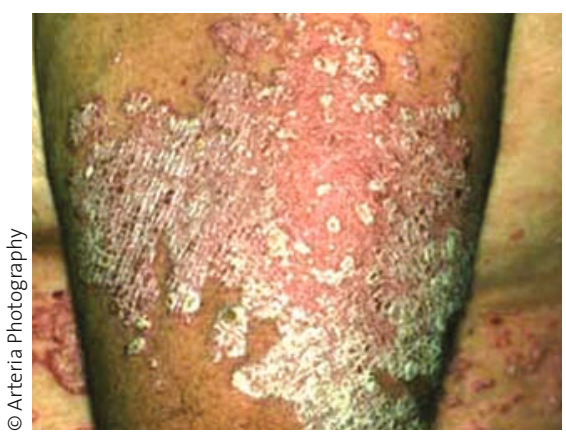

Eine ausgeprägte Schuppenflechte erhöht das kardiovaskuläre Risiko ebenso stark wie ein Diabetes mellitus.

- Psoriasispatienten leiden häufiger an Herzkrankheiten als Hautgesunde. Das genaue Ausmaß der Risikoerhöhung untersuchten Autoren der Universität Kopenhagen prospektiv anhand der gesamten dänischen Bevölkerung. In den Jahren 1997-2006 wurden sämtliche
Verschreibungen, Hospitalisierungen und Todesfälle registriert. Die Autoren identifizierten etwa 37000 Patienten mit leichten Formen der Schuppenflechte, knapp 3000 Patienten mit schwerer Psoriasis sowie 138000 Patienten mit Diabetes mellitus.

Mittels Multivarianzanalyse wurde das Risiko für Herzinfarkt, Schlaganfall oder kardiovaskulären Tod berechnet. Gegenüber Gesunden war es bei leichter Psoriasis um 10\%, bei schwerer Psoriasis um 30\% und bei Diabetes um 42\% erhöht. Die Gesamtsterblichkeit war nur bei Diabetes (um 39\%) und bei schwerer Psoriasis (um 52\%) erhöht. Fazit der Autoren: Bei Patienten mit Schuppenflechte sollten kardiovaskuläre Risikofaktoren konsequent behandelt werden.

$\mathrm{DE}=$

- Quelle: O. Ahlehoff et al., ESC-Jahreskongress 2010; Eur Heart J, Vol. 31, Abstract Suppl, \#3563
Selbst in Griechenland Mediterrane Diät ist bei Schülern out - Auf der Suche nach den Gründen für die zunehmende Fettleibigkeit der Jugend fanden Ärzte der Universität in Athen heraus, dass die junge Generation nicht mehr viel am Hut hat mit dem olympischen Geist und schon gar nicht mit der mediterranen Diät. Vor allem die Ernährungsgewohnheiten der untersuchten 500 Schulkinder schreien zum Himmel: Nur $6 \%$ konsumieren die gesunde mediterrane Kost, die Hälfte ernährt sich ausgesprochen ungesund. Bemerkenswert: Wer gesund $a ß$, trieb auch mehr Sport. Wer ungesund $a ß$, saß mehr vor Bildschirmen. Die Autoren empfehlen, in der Adipositas-Prävention bei Kindern auf Ernährungserziehung und mehr Bewegung zu setzen.

- D. Tsiachris, ESC-Jahreskongress 2010; Eur Heart J, Vol. 31, Abstr Suppl, \#5511 